\title{
DIELECTRIC RESPONSE OF RCN CRYSTAL AT ULTRA LOW FREQUENCIES
}

\author{
E.C. Ziemath and M.A. Aegerter \\ Instituto de Física e Química de São Carlos, Universidade de São Paulo, \\ 13560 São Carlos (SP) Brasil
}

(Received 31 January 1986 by G.F. Bassani)

The dielectric response of pure $\mathrm{KCN}$ crystals ( $\varepsilon^{\prime}, \varepsilon^{\prime \prime}$ and $\operatorname{tg} \delta$ ) has been measured as a function of temperature in the frequency range $10^{-2} \mathrm{~Hz}$ to $10^{4} \mathrm{~Hz}$. In the antiferroelectric phase the width of the loss peak are found practically independent of temperature ( 1.4 decades) and close to a Debye behavior; the relaxation time of the $\mathrm{CN}^{-}$dipoles is characterized by an Arrhenius behavior $\tau=\tau_{0} \exp (\mathrm{U} / \mathrm{KT})$ with $\tau_{0}^{\prime}=7.26 \times 10^{-15} \mathrm{~s}$ and $U=0,147 \mathrm{eV}$ confirming a classical temperature activated reorientation of the dipoles.

The study of the reorientation and collective ordering behavior of 1 inear diatomic ions in ionic solids is a field of great interest. Pure alkali cyanides $(\mathrm{NaCN}, \mathrm{KCN}, \mathrm{RbCN}$ and $\mathrm{CsCN}$ ) are among the many components which have been extensively investigated both experimentally and theoretically. The anisotropic properties of the $\mathrm{CN}^{-}$molecular ion are of two types:

a) an elastic dipole tensor due to the non spherical shape of the molecular ion.

b) an electric dipole vector due to the asymetric head and tail charge distribution of the molecule.

KCN crystallizes from the melt in the NaCl structure ${ }^{1,2}$. At high temperature anion sites have an octahedral pseudocubic symetry due to the fast reorientation of the $\mathbb{N N}^{-}$ions 3,4 . As the temperature is lowered this compound exhibits at $168 \mathrm{~K}$ a structural phase transition of first order to a body centered orthorhombic structure ${ }^{5,6}$. The $\mathrm{CN}^{-}$axis align along the $\langle 110\rangle$ directions of the original cubic structure but the sense of the ions do not. This means that the electric quadrupolar moment orders but the electric dipole moment does not; the $\mathrm{CN}^{-}$ions are still rapidly orienting head and tail. The order is purely elastic ${ }^{7}$. A second transition occurs at $83 \mathrm{~K}$ where the $\mathrm{CN}^{-}$ions become ordered in an antiferroelectric manner ${ }^{8-10}$. In this phase the head to tail reorientations of the $\mathrm{CN}^{-}$ions are still present but occur at a much lower rate which gradually diminishes with the temperature until obtainning a full ordering at $0 \mathrm{~K}$. The structure is primitive orthorhombic. Reorientation and ordering involve the rotation of the molecule within a rigid fixed lattice as well as sizeable deformations of the lattice due to strong anisotropic coupling of the $\mathrm{CN}^{-}$ with its surrouding.

The average time between the reorientation of $\mathrm{CN}^{-}$ions in alkali cyanides has been expeperimentally measured over eleven decades of value using MR technique ${ }^{11-13}$, dielectric techniques ${ }^{14-17}$ in the frequency range $50 \mathrm{~Hz}$ to $100 \mathrm{KHz}$ and ionic thermal conductivity (ITC) ${ }^{17}$, a pratically dc technique.

In this communication we present new data of the dielectric response of pure KCN single crystals measured in the frequency range $10^{-2}$ to $40 \mathrm{~Hz}$ as well as data for higher frequencies $^{18}$ confirming the previous results. The new data fill partly the frequency range between ITC and earlier high frequency measurements.

The dielectric response ( $\varepsilon^{\prime}, \varepsilon^{\prime \prime}$ and $\operatorname{tg} \delta$ ) has been measured at high frequency with a commercial capacitance bridge (General Radio model $1615-A)$. The response at low frequency was obtained with an equipment developed in our laboratory ${ }^{19-20}$. This equipment measures the current amplitude flowing through the sample $I=I_{0} e^{j(\omega t+\phi)}$ and its phase difference with the applied voltage $V=v_{0} e^{j \omega t}$ using modern di gital techniques; the frequency range is automatically scanned. The dielectric parameters are given by

$$
\begin{gathered}
\varepsilon^{\prime}=\frac{I_{0}}{\omega C_{0} V_{0}} \cos \delta \quad \quad E^{\prime \prime}=\frac{I_{0}}{\omega C_{0} V_{0}} \sin \delta \\
\operatorname{tg} \delta=\frac{\varepsilon^{\prime \prime}}{\epsilon^{\prime}}
\end{gathered}
$$

where $C_{0}$ is the geometrical capacitance of the sample $C_{0}=\varepsilon \frac{S}{d}$.

The crystals have been grown in our labo- 
boratory by the Czochralsky technique using Merck pro analysis material. The samples (area $15 \times 10 \mathrm{~mm}^{2}$ ) have been cleaved or cut in thin slices and then finely polished until obtaining thicknesses of the order of $d=0,4$ to $0,5 \mathrm{~mm}$. Gold electrodes with guard rings have been evaporated on both faces. The sample holder was designed such that the crystal is standing almost free in the cell in order to avoid mecanical stresses ${ }^{18}$. Miniature coaxial cable are soldered on the electrodes with silver paint. The measurements have been performed in a Supervaritemp Janis cryostat (helium gas cooling) where the crystal temperature is measured with a calibrated diode sensor and regulated to $\pm 0,1 \mathrm{~K}$.

Figure 1 shows the temperature behavior of the dielectric constant $\varepsilon^{\prime}$ measured at $1 \mathrm{KHz}$ (dotted line) and at lower frequencies: $~ 40$, $10,1,10^{-1}$ and $10^{-2} \mathrm{~Hz}$. All the data have been taken with decreasing temperatures. For $\mathrm{T}<250 \mathrm{~K}$

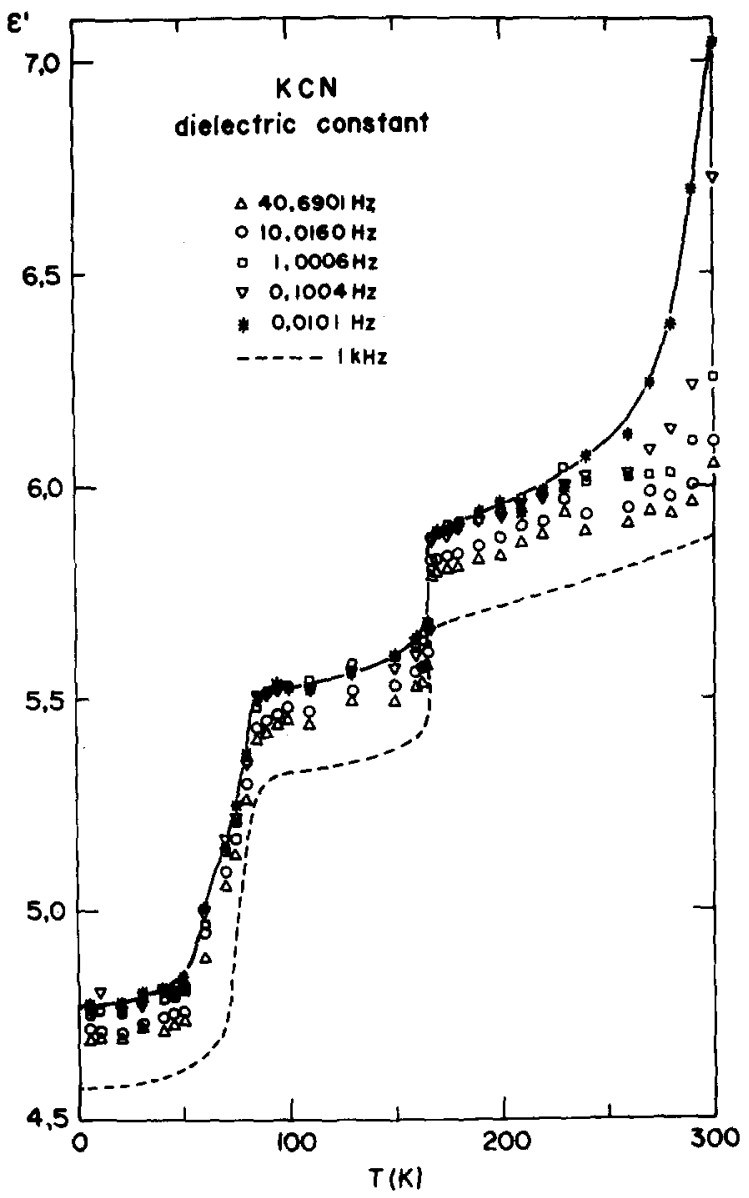

FIG. 1

Temperature dependence of the dielectric constant $\mathcal{E}^{\prime}$ of pure $\mathrm{KCN}$ measured at $40 \mathrm{~Hz}(\Delta)$, $10 \mathrm{~Hz}(\mathrm{O}), 1 \mathrm{~Hz}(\square), 10^{-1} \mathrm{~Hz}(\nabla)$ and $10^{-2} \mathrm{~Hz}$ (*) with our low frequency equipment. The dotted curve, shows as reference, has been obtained at $1 \mathrm{KHz}$ with a commercial bridge Genrad 6015-A. the curves appeared slightly shifted toward higher values of $\epsilon^{\prime}$ as the frequencies decrease. The same behavior has been observed for pure $\mathrm{KBr}$ crystals; this peculiar behavior are thought to arise from electronic difficulties in the precise calibration of the electrometer response as a function of the frequency ${ }^{18-20}$. The increase observed at low frequency for $\mathrm{T}>250 \mathrm{~K}$ is however a real effect of polarization which becomes important at extremely low frequencies already near room temperature 21 . The jump in $\varepsilon^{\prime}$ at the ferroelastic phase transition and which is not accompanied by a dielectric loss occurs always at the same temperature $T=168 \mathrm{~K}$ independently of the frequency of the applied field. It shows however a thermal hysteresis (not shown).

Figure 2 shows in more details the behavior of $\varepsilon^{\prime}$ and $\operatorname{tg} S$ in the antiferroelectric phase as a function of temperature measured for different frequencies $\left(10^{-2}\right.$ to $\left.10^{4} \mathrm{~Hz}\right)$. The curves
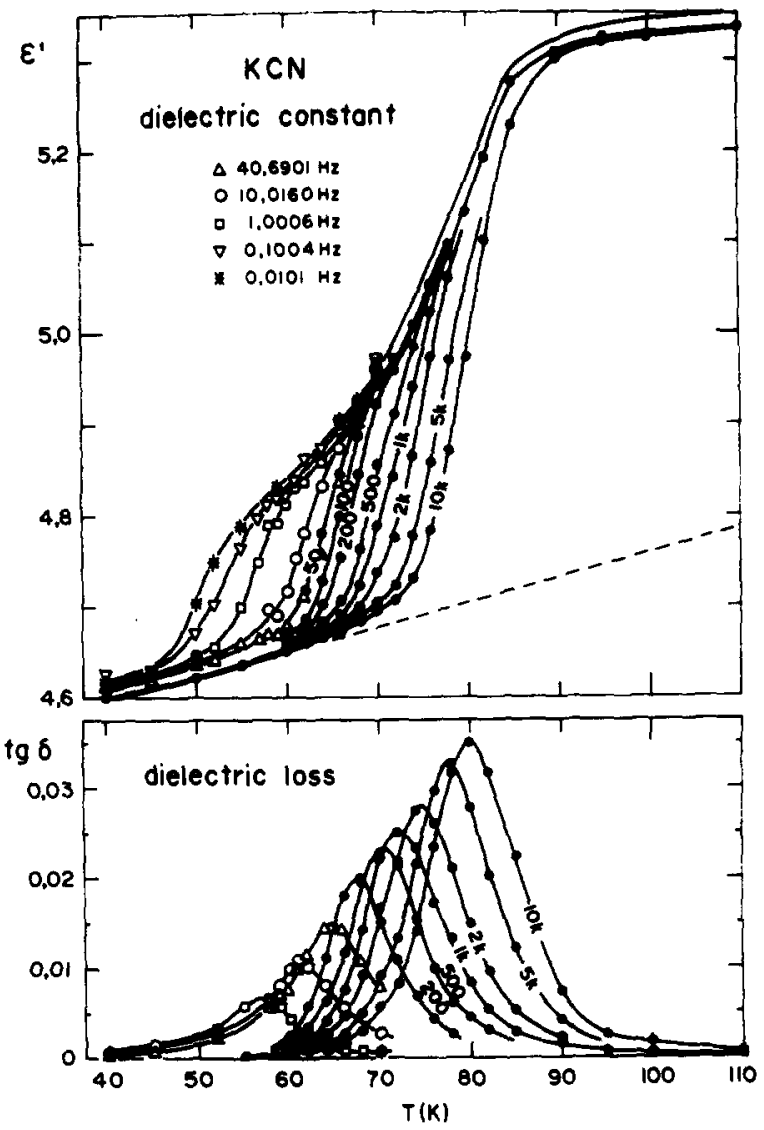

FIG. 2

Temperature dependence of the dielectric constant $\varepsilon^{\prime}$ and $\operatorname{tg} \delta$ of pure $\mathrm{KCN}$ in the antiferroelectric region measured at various frequencies $f=10^{-2}$ to $f=10^{4} \mathrm{~Hz}$. The curves have been slightly shiftedidownwards (see text). The dotted line is an extrapolation and represents the expected temperature variation of the dielectric constant at high frequencies $\varepsilon_{\infty}$ (see also fig.4). 
of $E^{\prime}$ have been slightly shifted downwards in order to practically coincide at $\mathrm{T}<40 \mathrm{~K}$. As the frequency diminishes we clearly observe a shift and a decrease of $\mathcal{E}^{\prime}$ and of the dielectric loss $\varepsilon^{\prime \prime}$ towards lower temperatures showing that the dipole still reorient even at lower temperatures.

The temperature dependence of the dielectric loss peak shift in frequency is shown in figure 3 . The data have been obtained from the

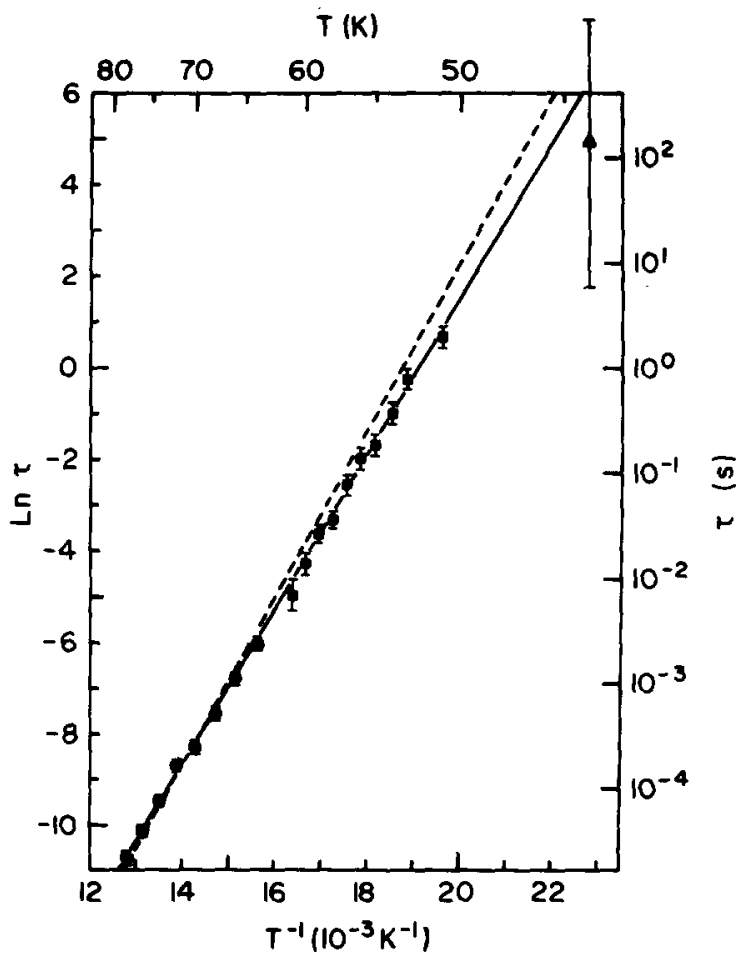

FIG. 3

Arrhenius plot of the relaxation time of $\mathrm{CN}^{-}$ dipoles versus inverse temperature $\tau=\tau{ }_{\mathrm{o}} \exp (\mathrm{U} / \mathrm{KT})$ with $\tau_{\mathrm{o}}=7.26 \times 10^{-15} \mathrm{~s}, \mathrm{U}=0,147 \mathrm{eV}$ obtained at high frequencies ( $\bullet$ ) (Genrad bridge) and low frequencies ( $\mathbf{c})$. The ITC result ( $\wedge$ ) is taken from reference 17. The bars represent the uncertainties of the measurements. The dotted line is the fit obtained by Lüty et al 17

maximum of the loss peaks. Also plotted in the figure is the result of the ionic thermal conductivity (ITC) technique obtained by ortizLope ${ }^{16}$. The temperature dependence is perfectly fitted to an Arrhenius law behavior $\tau=\tau_{0} \exp (U / K T)$ with $\tau_{0}=7.26 \times 10^{-15} \mathrm{~s}$ and $\mathrm{U}=0,147 \mathrm{eV}$ in good agreement with the values obtained by Lüty et al ${ }^{17}$ (dotted line). The shape of the peaks are close to that predicted by the Debye equations. Their width at half maximum is pratically constant in the temperature range studied; it varies only from 1.36 decades at $68 \mathrm{~K}$ to 1.44 decades at $53 \mathrm{~K}$ (to be compared with 1.144 decades for the Debye equation). This effect can be interpreted as being produced by a distribution of relaxation times such as the. Cole-Cole distribution proposed by Ortiz-Lopez ${ }^{16}$. The area under the $\varepsilon^{\prime \prime}$ curves in $\log _{10} w$ scale A (E") is directly related to the static susceptibility of the material

$\varepsilon_{s}-E_{\infty}=4 \pi X_{s}=1.466 \mathrm{~A}\left(\varepsilon^{\prime \prime}\right)$

where $\varepsilon_{s}$ and $\varepsilon_{\infty}$ are respectively the dielectric constant of the material measured at zero frequency $\varepsilon_{s}=\varepsilon^{\prime}(o)$ and at infinite frequency. This area decreases with decreasing temperatures and corresponds to a diminution in the number of the mobile $\mathrm{CN}^{-}$dipoles as a consequence of the gradual formation of the antiferroelectric ordered state with frozen-in electric dipoles. The difference between the curves $\xi^{\prime}\left(10^{-2} \mathrm{~Hz}\right.$ ) and $\varepsilon^{\prime}(\infty)$ (dotted line extrapolated in figure 2) is plotted in figure 4 with

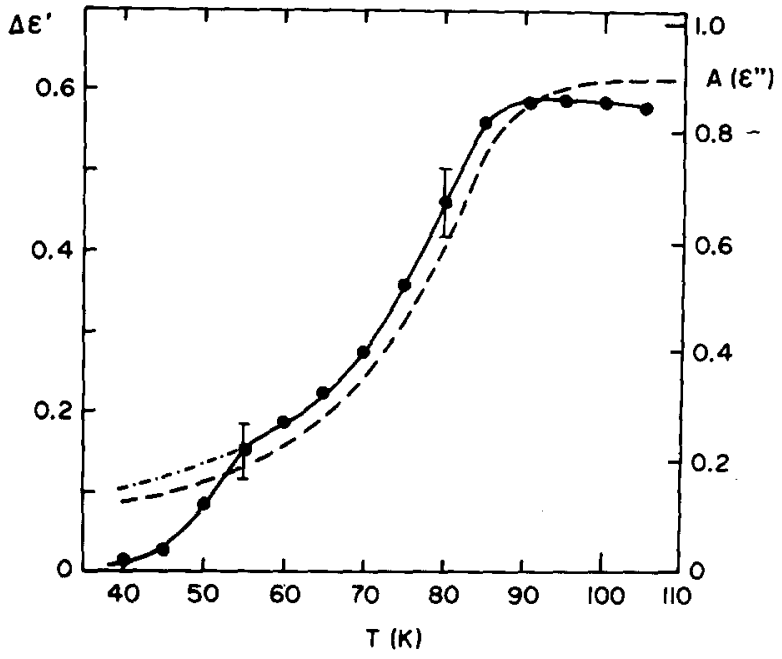

FIG. 4

Temperature dependence of the difference between the dielectric constant $\varepsilon^{\prime}$ measured at $10^{-2} \mathrm{~Hz}$ and at infinite frequency (dotted curve extrapolated of figure 2). For $T>55 \mathrm{~K}$ it represents the difference $\Delta \varepsilon=\varepsilon_{\mathbf{s}}-\epsilon_{\infty}=1.466 \mathrm{~A}\left(\varepsilon^{\prime \prime}\right)$ where $A\left(\varepsilon^{\prime \prime}\right)$ is the integrated loss area (dotted line as measured by Ortiz-Lopez ${ }^{16}$. The drop of $\varepsilon^{\prime}$ observed for $\mathrm{T}<55 \mathrm{~K}$ shows that the dipoles still not frozen-in cannot respond to the low frequency applied field $\left(10^{-2} \mathrm{~Hz}\right)$.

the results of Ortiz-Lopez ${ }^{16}$ for $A\left(\varepsilon^{\prime \prime}\right)$. The agreement is found reasonable for $T>55 \mathrm{~K}$ and confirms the model of random internal field with a simple block function distribution of local electric fields $P\left(E_{B}\right)$, symmetric with respect to $\mathrm{E}_{\mathrm{B}}=0$. Down to $55 \mathrm{~K}$ the curve $\varepsilon^{\prime}\left(10^{-2} \mathrm{~Hz}\right)$ is therefore a good approximation of the static value of the dielectric constant $\varepsilon^{\prime}(o)$. At 
$T=55 \mathrm{~K}$ most of the dipoles are frozen-in and the drop observed below this temperature reflects the fact that these dipoles still mobile cannot respond to the low frequency applied field $\left(10^{-2} \mathrm{~Hz}\right)$.
Acknowledgement - We acknowledge the financial support from the Fundação de Amparo à Pesquisa do Estado de São Paulo (FAPESP) and the Financiadora de Estudos e Projetos (FINEP).

\section{RriFliRlint:LS}

I. A. COOPliR, Nalure 107, 745 (1921).

2. R.M. Bozchitl, Journal of the American chemical Society 44, 317 (1922).

3. D.L. PRICE, J.M. ROWL, J.J. RUSIL, E. PRINLE, B, G. HINKS and S. SUSHAN, Jowrnal of Chemical Ihysics 56,3697 (1972).

4. J.M. ROHE, D.G. HINKS, D.L. PRINCE, S. SUSMAN and J.J. RUSH, Journal of bhemical physics 58 $2039(1973)$.

5. H.J. VERUEEL and J.M. BIJVOFT, Zeitschrift für Kristallografie 150, 201 (1978).

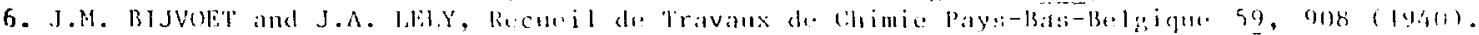

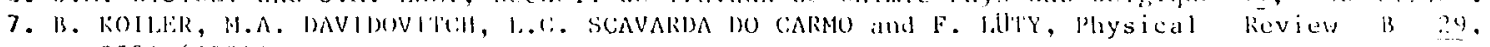
$3586(1984)$.

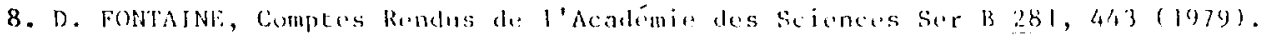

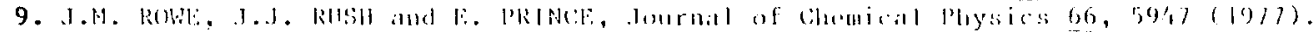

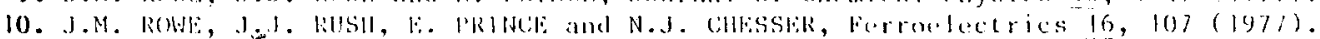

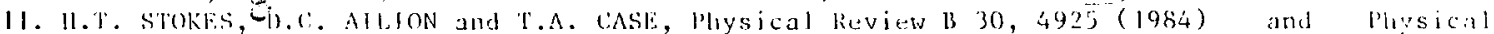
Review Letters 47,268 (1981)

12. H. BUCHEIT, S. ELSCHNER, H.D. MAIER, J. PETERSON and E, SCIHEIDER, Solid State Commenications $38,665(1981)$.

13. S. ELSCHAER and J. PETERSON, Zeitschrift fiir Physik B 52, 37 (1983).

14. M.D. IJIAAH, Ph. D. Thesis (1976) University of lltah (11.S.A.).

15. M.D. Jllbidid and F. LijTy, lierrowlectrics 16, 201 (1977).

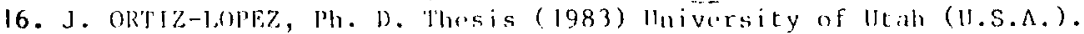

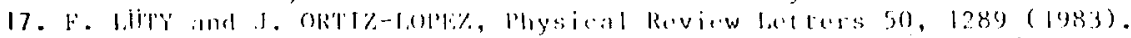

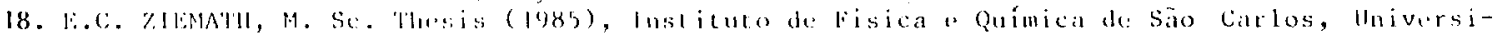
dade de sáo laule (Brasil).

19. J.F. Stakts. Ph. D. Thesis (19/9), Institulo de Física e Quimica de Säo Carlas, luiversida-

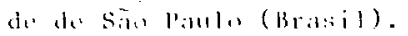

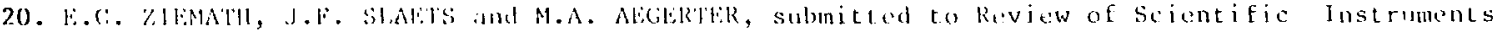
$(1986)$.

21. J.H. BEAMONT and P.W.M. JACOBS, lournal of Physics and Chemistry of Solids 28, 657 (1967). 